\title{
BMJ Open Pharmacologic and non-pharmacologic interventions to prevent hypersensitivity reactions of non-ionic iodinated contrast media: a systematic review protocol
}

To cite: Umakoshi $\mathrm{H}$, Nihashi T, Shimamoto $\mathrm{H}$, et al. Pharmacologic and nonpharmacologic interventions to prevent hypersensitivity reactions of non-ionic iodinated contrast media: a systematic review protocol. BMJ Open 2020;10:e033023. doi:10.1136/ bmjopen-2019-033023

- Prepublication history and additional material for this paper are available online. To view these files, please visit the journal online (http://dx.doi. org/10.1136/bmjopen-2019033023).

A preliminary work of this research project based on a brief literature search has been presented in an educational session at the 77th annual meeting of the Japan Radiological Society 2018, Yokohama, Japan.

Received 17 July 2019 Revised 11 February 2020 Accepted 12 February 2020

Check for updates

(C) Author(s) (or their employer(s)) 2020. Re-use permitted under CC BY-NC. No commercial re-use. See rights and permissions. Published by BMJ.

For numbered affiliations see end of article.

Correspondence to Dr Teruhiko Terasawa; terasawa@fujita-hu.ac.jp

\section{ABSTRACT}

Introduction lodinated contrast media are commonly used in medical imaging and can cause hypersensitivity reactions, including rare but severe life-threatening reactions. Although several prophylactic approaches have been proposed for severe reactions, their effects remain unclear. Therefore, we aim to review systematically the preventive effects of pharmacologic and non-pharmacologic interventions and predictors of acute, hypersensitivity reactions.

Methods and analysis We will search the PubMed, EMBASE and Cochrane Central Register of Controlled Trials databases from 1 January 1990 through 31 December 2019 and will examine the bibliographies of eligible studies, pertinent review articles and clinical practice guidelines. We will include prospective and retrospective studies of any design that evaluated the effects of pharmacological and non-pharmacological preventive interventions for adverse reactions of non-ionic iodinated contrast media. Two assessors will independently extract the characteristics of the study and intervention and the quantitative results. Two independent reviewers will assess the risk of bias using standard design-specific validity assessment tools. The primary outcome will be reduction in acute contrast media-induced hypersensitivity reactions. The secondary outcomes will include characteristics associated with the development of contrast mediainduced acute hypersensitivity reactions, and adverse events associated with specific preventive interventions. Unique premedication regimens (eg, dose, drug and duration) and non-pharmacological strategies will be analysed separately. Average-risk and high-risk patients will be considered separately. A meta-analysis will be performed if appropriate.

Ethics and dissemination Ethics approval is not applicable, as this will be a secondary analysis of publicly available data. The results of the analysis will be submitted for publication in a peer reviewed journal.

PROSPERO registration number CRD42019134003
Strengths and limitations of this study

- This will be the first systematic review and metaanalysis to assess and compare the preventive effectiveness of pharmacologic and non-pharmacologic interventions for preventing acute hypersensitivity reactions caused by non-ionic iodinated contrast media.

- Comprehensive literature searches and up-to-date systematic review methodologies will be used to identify actionable evidence.

- If the number of studies is too small, or clinical or statistical across-study heterogeneity is deemed too great, a quantitative synthesis may not be feasible.

\section{INTRODUCTION}

Iodinated contrast media are commonly used to enhance CT examinations for diagnosis and treatment monitoring. However, non-ionic iodinated contrast media cause adverse reactions ranging from mild nausea or pruritus to haemodynamic shock and cardiopulmonary arrest in approximately $3 \%$ of patients. ${ }^{12}$ Life-threatening reactions occur in approximately 4 in 10000 cases. ${ }^{1}$ As millions of doses of iodinated contrast media are administered annually, severe reactions are expected to occur commonly within a population. ${ }^{3}$

The mechanism underlying adverse reactions induced by contrast media is not fully understood and is likely multifactorial. ${ }^{2}$ However, based on a general framework for the classification of adverse drug reactions, the reactions induced by contrast media can be divided into two types-commonly referred to as type A and type B reactions. ${ }^{45}$ Type A reactions are physiologic and often dose-dependent reactions that are expected 
from the pharmacologic properties of the administered contrast media. Type B reactions are hypersensitivity reactions that are neither physiologic nor dose-dependent, and are usually unpredictable. Distinction between type A and type $\mathrm{B}$ reactions can facilitate designing prophylactic strategies for preventing contrast media-induced adverse reactions; nevertheless, the distinction is not straightforward, and some professional societies have discordant classification systems. ${ }^{4}$

No perfect strategy has been established to mitigate the risk of acute severe contrast media-induced hypersensitivity reactions. Only weak evidence supports pharmacological interventions including corticosteroids and/or antihistamines. ${ }^{2}$ For example, premedication often fails ${ }^{6}$ and can induce adverse effects such as corticosteroidinduced hyperglycaemia and indirectly contributed to prolonged hospitalisation. ${ }^{78}$ Purported risk factors for contrast media-induced reactions predict reactions of any severity; they do not specifically predict acute lifethreatening reactions. ${ }^{29}$ Further, the comparative effectiveness of alternative preventive strategies involving pharmacological and non-pharmacological interventions has not been systematically evaluated. ${ }^{6-8}$ 10-12 Although professional societies including the American College of Radiology (ACR) propose several premedication regimens, ${ }^{2}$ only one has been tested in a randomised design, and that study had methodological challenges. ${ }^{13}$ Premedication practice varies, ${ }^{14} 15$ which precludes a standardised comparative assessment among alternative pharmacological and non-pharmacological interventions. Given this uncertainty, the 2019 European Society of Urogenital Radiology (ESUR) Guideline on Contrast Agents indicates that "premedication is not recommended because there is not good evidence of its effectiveness (page 7, A1.1). ${ }^{9}$

Since the publication of two systematic reviews in 2006 that evaluated the effectiveness of premedication regimens, ${ }^{16}{ }^{17}$ several relevant studies of pharmacological and alternative, non-pharmacological strategies (eg, exchanging one contrast medium for an alternative) have been published and have influenced the ACR and ESUR guidelines. ${ }^{18} 19$ In addition, the two prior systematic reviews on this topic included pharmacological prophylaxis only in the context of now-outdated high-osmolality iodinated contrast media that are no longer used in clinical practice. Therefore, we planned a comprehensive quantitative synthesis of clinical data on the effects of pharmacological and non-pharmacological prophylactic strategies for the prevention of acute adverse reactions to non-ionic iodinated contrast media.

\section{METHODS AND ANALYSIS}

This systematic review protocol follows the Preferred Reporting Items for Systematic Review and Meta-Analysis Protocols 2015 statement. ${ }^{20}$ Based on the analytic framework shown in figure 1, we have formulated the following three key research questions and related subquestions:

\section{Key question 1}

What is the effect of interventions to reduce acute $(<1$ hour) hypersensitivity (type B) reactions in patients receiving contrast media?

Key question 1a: What is the preventive effect of guideline-recommended oral (12 or 13hours), guideline-recommended accelerated intravenous

Key Question 1. What is the effect of interventions to reduce acute ( $<1$ hour) hypersensitivity (Type B) reactions in patients receiving CM?

Key Question 2. What are the patient-level and intervention-level characteristics (i.e., predictors) associated with CM-induced acute hypersensitivity (Type B) reactions?

Key Question 3. What are the complications and adverse events associated with specific interventions to reduce CM-induced adverse reactions?

Interventions/Comparators:

Pharmacological interventions

-12- or 13-hour corticosteroids with or without anti-histamine

-5- to 11-hour accelerated IV corticosteroids with or without anti-histamine

-Any preps less than 5 hours, including

steroids, anti-histamine, or both

Non-pharmacological interventions

-Switching agents

-Other interventions

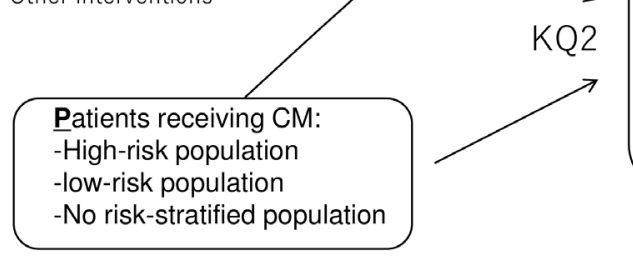

Predictors of clinical outcomes

Patient-level characteristics

-Prior adverse reactions

-Allergic diathesis

Intervention-level characteristics

-Type (and dosing) of interventions

(premedication drugs)

-Patterns of CM change

Figure 1 Analytic framework. CM, contrast media; KQ, key question. 
(5-11 hours) or non-guideline emergent intravenous ( $<5$ hours) premedication on acute $(<1$ hour) hypersensitivity reactions in patients receiving contrast media?

Key question 1b: What is the preventive effect of a change of contrast media alone on acute $(<1$ hour) hypersensitivity reactions in patients receiving contrast media?

Key question $1 \mathrm{c}$ : What is the preventive effect of combining standard oral (12 or 13 hours) premedication and a change of contrast media on acute $(<1$ hour) hypersensitivity reactions in patients receiving contrast media?

Key question 1d: What is the preventive effect of other interventions (other than the above listed) on acute $(<1$ hour) hypersensitivity reactions?

Key question 1e: What is the preventive effect of any interventions for acute $(<1$ hour) adverse reactions of any type (ie, both type A and B reactions)?

\section{Key question 2}

What are the patient-level and intervention-level characteristics (ie, predictors) associated with contrast mediainduced acute hypersensitivity (type $\mathrm{B}$ ) reactions?

\section{Key question 3}

What are the complications and adverse events associated with specific interventions to reduce contrast mediainduced adverse reactions?

\section{Literature search}

We will search the PubMed, EMBASE and Cochrane Central Register of Controlled Trials databases from 1 January 1990 through 31 December 2019 for both Englishlanguage and non-English-language publications, using search terms such as 'iodinated contrast media', 'premedication', 'adverse reaction', 'breakthrough reactions' and their synonyms. The complete search strategy and full list of databases are available in online supplementary file. We will include studies published after 1990, when nonionic contrast media were developed and disseminated widely. We also will examine the references of eligible studies, relevant review articles and existing clinical practice guidelines developed by professional societies such as the ACR and ESUR. ${ }^{29}$ All potentially eligible non-English publications will be translated into English before fulltext assessment.

\section{Inclusion and exclusion criteria}

We will include studies that assessed patients who received intravenous or intra-arterial non-ionic iodinated contrast media and any interventions to reduce contrast media-induced adverse reactions. Table 1 presents our detailed inclusion criteria, which follow a generally accepted framework to formulate a systematic review question comprising five key components: populations, interventions, comparator interventions, outcomes and study designs. ${ }^{21}$ Regarding pharmacological prophylactic interventions, we will focus on premedication based on corticosteroids, antihistamines, or both, and exclude studies that tested other medications (eg, ephedrine, diazepam, atropine) because these are not relevant to current clinical practice. We also will exclude studies that assessed patients who received highosmolality contrast media because they are no longer used in clinical practice. Both prospective and retrospective studies of any design that evaluated at least 10 patients will be included.

Several frameworks for categorising clinical symptoms and severity induced by pharmacological agents including contrast media exist. We will employ an accepted general two-group framework (type A and type B) to classify acute contrast media-induced adverse reactions reported in primary studies in the main analysis. ${ }^{5}$ We then will reclassify the reported acute adverse reactions using the current ACR categorisation system ${ }^{2}$ in a sensitivity analysis to assess the applicability and difference between the two frameworks. Delayed reactions occurring more than 1 hour after contrast media administration will not be assessed. A breakthrough reaction will be defined as an acute type B reaction of any severity that occurs despite premedication. We will operationally classify any randomised controlled trials (RCTs) and any studies with a non-randomised design that compared two or more intervention groups (ie, so-called non-randomised studies of intervention (eg, quasi-RCTs, cohort studies, case-control studies)) as 'comparative studies.' 'Noncomparative studies' will include single-group studies and case series.

We will exclude editorials, comments, letters to the editor and review articles. Multiple publications with potentially overlapping patient populations can overestimate the volume of evidence. Therefore, for overlapping study populations, we will only include the publication with the largest sample size. We will contact the study authors by email if the publications do not report adequate information about the patient characteristics and reaction classifications. We will consider our request to be rejected if two email request reminders sent separately 14 days after the initial contact attempt are not returned.

The results of our electronic searches will be imported into reference management software and duplicate results will be removed. Multiple paired investigators will independently double-screen non-overlapping sets of abstracts (eg, the first half of the abstracts will be assigned to team A (two investigators) and the second half of the abstracts will be assigned to team B (two investigators) in the case of two paired teams) and examine full-text articles for potentially eligible citations. We will use Abstrackr (Center for Evidence Synthesis in Health, Brown University, available at abstrackr.cebm.brown. edu), a free, open-source, citation screening programme for abstract screening. A third investigator will adjudicate any discrepant results if consensus cannot be reached between the two reviewers. 
Table 1 Inclusion criteria based on the PICOD framework

\begin{tabular}{ll} 
PICOD & Specific details \\
\hline Population & Patients who received intravenous or intra-arterial non-ionic iodinated $\mathrm{CM}^{*}$ \\
& High-risk population \\
& Low-risk population \\
& No risk-stratified population
\end{tabular}

Interventions / Comparators and co-interventions

Pharmacological interventions†

- 12 or 13 hours oral corticosteroids with or without antihistamine

- 5-11 hours IV corticosteroids with or without antihistamine

- Any premedication less than 5 hours using corticosteroids, antihistamine or both

Non-pharmacological interventions

- Change of CM that caused prior type B hypersensitivity reaction

\begin{tabular}{|c|c|}
\hline Outcomes & $\begin{array}{l}\text { Rates of acute ( }<1 \text { hour) type } B \text { hypersensitivity reactions } \neq \\
\text { Acute reaction-related deaths within } 30 \text { days } \\
\text { Severe reactions only } \\
\text { Moderate and severe reactions only } \\
\text { Upgraded reactions compared with the index reactions } \\
\text { All hypersensitivity reactions }\end{array}$ \\
\hline $\begin{array}{l}\text { Predictors of acute adverse } \\
\text { reactions }\end{array}$ & $\begin{array}{l}\text { Patient-level characteristics } \\
\text { Prior type B hypersensitivity reactions } \\
\text { - Prior type A physiologic reactions§ } \\
\text { Allergic diathesis (eg, asthma, food or drug allergy, etc) } \\
\text { Intervention-level characteristics } \\
\text { Types and regimens of interventions } \\
\text { Dosing of specific premedication drugs } \\
\text { - Change of CM (specific class/product and/or dosing) }\end{array}$ \\
\hline
\end{tabular}

${ }^{*}$ Per-study defined risk criteria are allowed.

†Both guideline-recommended and ad-hoc regimens are allowed, but will be analysed separately. Guideline-recommended oral regimens are defined as follows ${ }^{2}$ : 13 hours regimen: prednisone $50 \mathrm{mg} \mathrm{PO}$ at 13,7 and 1 hours before CM injection+/-optional diphenhydramine $50 \mathrm{mg}$ IV, $\mathrm{IM}$ or PO at 1 hour before CM injection; 12 hours regimen: methylpredonisolone $32 \mathrm{mg} \mathrm{PO}$ at 12 and 2 hours before CM injection+/-optional antihistamine. Guideline-recommended urgent regimens are: methylprednisolone $40 \mathrm{mg}$ or hydrocortisone $200 \mathrm{mg}$ IV every 4 hours until CM injection (minimum cumulative duration 5 hours)+/-diphenhydramine $50 \mathrm{mg}$ IV at 1 hour before CM injection. Any premedication that does not include corticosteroids or that is less than 5 hours in duration is non-standard.

$\ddagger$ Grades of type B hypersensitivity reactions are defined as follows ${ }^{2}$ : mild reactions include limited urticaria/pruritus, cutaneous oedema, limited 'itchy'/'scratchy' throat, nasal congestion, sneezing, conjunctivitis and rhinorrhea; moderate reactions include diffuse urticaria/pruritus, diffuse erythema with stable vital signs, facial oedema without dyspnoea, throat tightness or hoarseness without dyspnoea, and wheezing/ bronchospasm with mild or no hypoxia; and severe reactions include diffuse oedema, facial oedema with dyspnoea, diffuse erythema with hypotension, laryngeal oedema with stridor and/or hypoxia, wheezing/bronchospasm with significant hypoxia and anaphylactic shock (hypotension+tachycardia).

§Grades of type A physiologic reactions are defined as follows ${ }^{2}$ : mild reactions include limited nausea/vomiting, transient flushing, warmth, chills, headache, dizziness, anxiety, altered taste, mild hypertension and vasovagal reaction that resolves spontaneously; moderate reactions include protracted nausea/vomiting, hypertensive urgency, isolated chest pain and vasovagal reaction that requires and is responsive to treatment; and severe reactions include vasovagal reaction resistant to treatment, arrhythmia, convulsions, seizures and hypertensive emergency.

$\mathrm{CM}$, contrast medium; IM, intramuscularly; IV, intravenously; PICOD, populations, interventions, comparator interventions, outcomes and study designs; $\mathrm{PO}$, orally.

\section{Data extraction}

We will extract the following descriptive data from eligible studies. Study characteristics will include first author, year of publication, journal and study design (prospective vs retrospective, comparative study vs noncomparative study). Participant characteristics will include age, sex, history and severity and type of any prior acute adverse reaction to iodinated contrast media, allergic diathesis including severe allergy(-ies) to other substances and asthma, ${ }^{2}$ and other known risk factors for adverse reactions. Contrast media characteristics will include brand and generic names and doses of contrast 
media administered. Intervention characteristics will include premedication strategies including drugs, doses, duration and change in contrast media. Outcome characteristics will include details of and change in adverse reactions (kinds and severity), assessors of adverse reactions (number and experience), and categorisation system to classify and grade acute adverse events. We will operationally define guideline-recommended regimens as the 12-13 hours oral administration of corticosteroids with or without use of an antihistamine, and standard accelerated regimen as a $5-11$ hours intravenous administration of corticosteroids with or without the use of an antihistamine. ${ }^{2}$ If a study adopted ad-hoc definitions or categorisation systems other than the two-group classification framework or those proposed by the ACR, we will specify these differences in sufficient detail. One primary investigator will extract the descriptive data, which will be verified by a second investigator.

Two reviewers will independently double-extract quantitative data from each publication. We will determine the relative risk of a hypersensitivity reaction between two (or more) groups in comparative studies. We will extract the number of patients in each group, as well as the number of patients who developed a hypersensitivity reaction. If relevant count data cannot be determined from the publication, we will instead extract the reported point estimates and their confidence intervals.

We will extract quantitative measures (eg, risk ratios, ORs) of the association of the presence or absence of a predictor with the development of a breakthrough reaction. We will prefer adjusted values over unadjusted values if both are reported. A priori candidate predictors selected for extraction include specific index type B reactions and their grades, and any allergic diathesis and its severity.

\section{Assessment of risk of bias}

For RCTs, we will use the revised tool to assess risk of bias in randomised trials (RoB 2 tool) ${ }^{22}$ We will assess five domains of RCT study validity (ie, randomisation process, deviations from intended interventions, missing outcome data, measurement of outcomes, selective reporting) and then assign an overall risk of bias for each trial.

For non-randomised intervention studies, we will use the Risk Of Bias In Non-randomised Studies of Interventions tool for cohort studies, ${ }^{23}$ and the Cochrane Risk Of Bias Assessment Tool for Non-Randomised Studies of Interventions for case-control studies. ${ }^{24}$ We will assess seven domains of study validity (ie, confounding, participant selection, classification of interventions, deviations from intended interventions, missing data, measurement of outcomes and selective reporting) and then assign an overall risk of bias for each study.

For single-group observational studies that assessed a predictor in a specific clinical context (eg, development of a breakthrough reaction under a premedication regimen), we will use a revised version of the Quality in Prognosis Studies tool (the QUIPS-2) ${ }^{25}$ We will assess six domains of study validity (study participation, study attrition, prognostic factor measurement, outcome measurement, confounding measurement and account, and analysis and reporting) and then assign an overall risk of bias for each study.

Two reviewers will independently assess each item and rate the domain-specific and overall risks of bias. Discrepant ratings will be resolved by consensus. A third independent investigator will adjudicate any unresolved discrepancies. The complete list of modified operational definitions used to rate each item will be available from the authors on request.

\section{Data synthesis}

The primary outcome of interest will be the relative risk of an acute type B (hypersensitivity) reaction between specific prevention strategies. Secondary outcomes will include the breakthrough reaction rate of each specific strategy and the predictive performances of covariates for overall and severe breakthrough reactions. For all outcome measures, we will first construct an evidence map by performing qualitative syntheses based on graphs and tables to examine the diversity and volume of available evidence on this topic. ${ }^{26}{ }^{27}$ If feasible, we will then perform a quantitative synthesis.

For summary relative measures (eg, relative risk of an acute type $\mathrm{B}$ reaction) based on count data, we will perform a random-effects meta-analysis using the binomial likelihood with logit link in a generalised linear modelling framework (ie, random-effects logistic regression).$^{28}$ If already-estimated relative measures are the only extractable formats, we will utilise the log-transformed estimates and their variances as 'plug-in' estimates. If appropriate, the meta-analytical model for a specific pairwise comparison will be extended to a network metaanalysis to synthesise data from both direct and indirect comparisons of all available studies in a single analysis. ${ }^{28}$

For summary estimates of the proportion measures in non-comparative studies, we will perform a randomeffects meta-analysis of proportions using the binomial likelihood and logit link (ie, so-called the binomialnormal model). ${ }^{29}$

\section{Additional analyses}

We will estimate the between-study SD parameter, tau, and $\mathrm{I}^{2}$ statistic and corresponding $95 \%$ credible intervals as measures of statistical heterogeneity. An $\mathrm{I}^{2}>50 \%$ will indicate intermediate heterogeneity, while an $\mathrm{I}^{2}>70 \%$ will indicate high heterogeneity. ${ }^{30}$

To explore statistical heterogeneity, we will perform subgroup analyses and, if feasible, a univariable randomeffects meta-regression. ${ }^{28}$ Preplanned candidate factors will include the use of guideline-recommended premedication regimens (vs non-guideline-recommended or ad-hoc regimens), alterations of the culprit contrast media (vs not), use of the general two-group classification framework versus the ACR categorisation systems for the classification and grading of reactions (vs others), and 
severity and type of prior reactions to iodinated contrast media. We will consider conducting sensitivity analyses by reclassifying and/or re-grading the reported reactions based on the two-group classification system and the ACR classification system for studies not using these classification frameworks, if pertinent individual-level data are presented.

We will assess funnel-plot asymmetry if at least 10 studies are included. ${ }^{31}$ To address potential biases derived from missing outcome data, we will apply the approach proposed by Turner et al. ${ }^{32}$ We will assess the certainty of evidence using the Grading of Recommendations Assessment, Development, and Evaluation approach. ${ }^{33}$

\section{Statistical software}

We will conduct all analyses using Stata V.14/SE (Stata Corp.) and OpenBUGS V.3.2.3 (members of OpenBUGS Project Management Group; see www.openbugs.net). All tests will be two-sided, and statistical significance will be defined as a $p$ value $<0.05$.

\section{Patient and public involvement}

We did not involve patients or the public in the preparation of this systematic review protocol.

\section{DISCUSSION}

The revised 2019 ESUR guidelines on contrast agents retracted recommendations for the premedication of patients at an increased risk of contrast reaction due to a lack of scientific evidence of efficacy. ${ }^{9}$ This position is inconsistent with the latest guidelines of other professional societies, including the ACR (ACR Manual on Contrast Media V.10.3), ${ }^{2}$ the Canadian Association of Radiologists ${ }^{34}$ and the Japan Radiological Society. ${ }^{35}$ Also, concerns have been raised on the relevance and impact of the classification systems and nomenclature of contrast media-induced adverse reactions, and their recommended management proposed in guidelines. ${ }^{4}$ Given the wide application of iodinated contrast media in medical imaging and interventional procedures, the uncertainty surrounding the optimisation of prevention strategies based on the proposed framework, and the absence of recently published evidence reviews, we believe that it will be worthwhile to conduct a new systematic review that critically examines the existing evidence on interventions to reduce acute contrast media-induced adverse reactions. Using a comprehensive evidence map of the published literature on the effects of pharmacologic and non-pharmacologic interventions and, if feasible, new meta-analytic results, we hope to clarify the actionable evidence regarding the use of preventive interventions.

\section{ETHICS AND DISSEMINATION}

The findings from the review will be disseminated through publications in peer-reviewed journals, and presentations at conferences.
Author affiliations

${ }^{1}$ Department of Radiology, Toyohashi Municipal Hospital, Toyohashi, Aichi, Japan ${ }^{2}$ Department of Radiology, Komaki City Hospital, Komaki, Aichi, Japan

${ }^{3}$ Department of Radiology, Nagoya University Graduate School of Medicine, Nagoya, Aichi, Japan

${ }^{4}$ Departments of Radiology and Urology, Michigan Medicine, Ann Arbor, Michigan, USA

${ }^{5}$ Department of Emergency and General Internal Medicine, Fujita Health University, Toyoake, Aichi, Japan

Contributors $\mathrm{HU}$ and TN originated the idea; HU, TN and TT drafted the initial version of the protocol; HU, TN and TT developed the search strategy; HS, TY, HI, $\mathrm{AT}, \mathrm{NH}, \mathrm{SI}, \mathrm{YT}, \mathrm{SN}$ and $\mathrm{MD}$ reviewed the protocol and suggested amendments. All authors read and approved the final version of the protocol. HU, TN and TT are guarantors of the review.

Funding TT was supported in part by the Ministry of Education, Culture, Sports, Science and Technology, Japan (JSPS KAKENHI Grant numbers: 26460755 and 19K07877).

Disclaimer The funding source had no role in the design or conduct of the study; collection, management, analysis and interpretation of the data; preparation, review or approval of the manuscript; and decision to submit the manuscript for publication.

Competing interests For complete transparency outside the submitted work, SI reports personal fees from Bayer Yakuhin, Ltd.; YT is an endowed chair sponsored by HIMEDIC Co. He does not receive any financial support from the corporation for conducting the research and writing this paper. He also reports personal fees from Daiichi Sankyo company, Ltd., Bayer Yakuhin, Ltd., GE Healthcare, Medi-Physics Co Ltd., Mitsubishi Tanabe Pharma Corporation, National Cancer Center; SN reports personal fees from Daiichi Sankyo Company, Ltd., Kowa Company, Ltd., Bayer Yakuhin, Ltd., Fuji Pharma Co Ltd., Bracco-Eisai Co Ltd., FUJIFILM Toyama Chemical Co Ltd., Canon Medical Systems Corporation, Siemens Healthineers Japan, Trust Clinic, Nagoya Jhohoku Radiology Clinic, Gakken Medical Shujunsha Co Ltd, Neuryon AG; and MD reports royalties from Wolters Kluwer and uptodate.com.

Patient consent for publication Not required.

Ethics approval As this is a systematic review, we are not planning to obtain a formal ethical approval.

Provenance and peer review Not commissioned; externally peer reviewed.

Open access This is an open access article distributed in accordance with the Creative Commons Attribution Non Commercial (CC BY-NC 4.0) license, which permits others to distribute, remix, adapt, build upon this work non-commercially, and license their derivative works on different terms, provided the original work is properly cited, appropriate credit is given, any changes made indicated, and the use is non-commercial. See: http://creativecommons.org/licenses/by-nc/4.0/.

ORCID iD

Teruhiko Terasawa http://orcid.org/0000-0002-0975-391X

\section{REFERENCES}

1 Katayama H, Yamaguchi K, Kozuka T, et al. Adverse reactions to ionic and nonionic contrast media. A report from the Japanese Committee on the safety of contrast media. Radiology 1990;175:621-8.

2 ACR Committee on Drugs and Contrast Media. ACR manual on contrast media version 10.3, 2018. Available: https://www.acr.org/-/ media/ACR/files/clinical-resources/contrast_media.pdf [Accessed 1 Jul 2019].

3 Bettmann MA. Frequently asked questions: iodinated contrast agents. Radiographics 2004;24(Suppl 1):S3-10.

4 Böhm I, Morelli J, Nairz K, et al. Myths and misconceptions concerning contrast media-induced anaphylaxis: a narrative review. Postgrad Med 2017;129:259-66.

5 Pichler WJ. Drug hypersensitivity: classification and clinical features. UpToDate. Available: https://www.uptodate.com/contents/drughypersensitivity-classification-and-clinical-features [Accessed 1 Jan 2020].

6 Davenport MS, Cohan RH, Caoili EM, et al. Repeat contrast medium reactions in premedicated patients: frequency and severity. Radiology 2009;253:372-9. 
7 Davenport MS, Cohan RH, Khalatbari S, et al. Hyperglycemia in hospitalized patients receiving corticosteroid premedication before the administration of radiologic contrast medium. Acad Radiol 2011:18:384-90.

8 Davenport MS, Mervak BM, Ellis $\mathrm{JH}$, et al. Indirect cost and harm attributable to oral 13-Hour inpatient corticosteroid prophylaxis before contrast-enhanced CT. Radiology 2016;279:492-501.

9 European Society of Urogenital Radiology. ESUR guidelines on contrast media ver10.0, 2018. Available: http://www.esur.org/ guidelines/en/index.php\#a [Accessed 1 Jul 2019].

10 Mervak BM, Davenport MS, Ellis JH, et al. Rates of breakthrough reactions in inpatients at high risk receiving premedication before contrast-enhanced CT. AJR Am J Roentgenol 2015;205:77-84.

11 Freed KS, Leder RA, Alexander C, et al. Breakthrough adverse reactions to low-osmolar contrast media after steroid premedication. AJR Am J Roentgenol 2001;176:1389-92.

12 Mesurolle B, Ariche M, Cohen D. Premedication before i.v. contrastenhanced CT resulting in steroid-induced psychosis. AJR Am J Roentgenol 2002;178:766-7.

13 Lasser EC, Berry CC, Mishkin MM, et al. Pretreatment with corticosteroids to prevent adverse reactions to nonionic contrast media. AJR Am J Roentgenol 1994;162:523-6.

14 O'Malley RB, Cohan RH, Ellis $\mathrm{JH}$, et al. A survey on the use of premedication prior to iodinated and gadolinium-based contrast material administration. J Am Coll Radiol 2011;8:345-54.

15 The Japan Radiological Society. A nationwide survey on the use of premidication prior to iodinated contrast material administration, 2015. Available: http://www.radiology.jp/content/files/20150721-2 pdf [Accessed 1 Jan 2020].

16 Delaney A, Carter A, Fisher M. The prevention of anaphylactoid reactions to iodinated radiological contrast media: a systematic review. BMC Med Imaging 2006;6:2.

17 Tramèr MR, von Elm E, Loubeyre $P$, et al. Pharmacological prevention of serious anaphylactic reactions due to iodinated contrast media: systematic review. BMJ 2006;333:675.

18 Abe S, Fukuda H, Tobe K, et al. Protective effect against repeat adverse reactions to iodinated contrast medium: premedication vs. changing the contrast medium. Eur Radiol 2016;26:2148-54.

19 Park HJ, Park J-W, Yang M-S, et al. Re-exposure to low osmolar iodinated contrast media in patients with prior moderate-to-severe hypersensitivity reactions: a multicentre retrospective cohort study. Eur Radiol 2017;27:2886-93.

20 Shamseer L, Moher D, Clarke M, et al. Preferred reporting items for systematic review and meta-analysis protocols (PRISMA-P) 2015: elaboration and explanation. BMJ 2015;350:g7647.

21 Counsell C. Formulating questions and locating primary studies for inclusion in systematic reviews. Ann Intern Med 1997:127:380-7.
22 Higgins JPT, Sterne JAC, Savović J. A revised tool for assessing risk of bias in randomized trials. In: Chandler J, McKenzie J, Boutron I, eds. Cochrane methods Cochrane database of systematic reviews, 2016. https://sites.google.com/site/riskofbiastool/welcome/rob-2-0tool?authuser=0

23 Sterne JA, Hernán MA, Reeves BC, et al. ROBINS-I: a tool for assessing risk of bias in non-randomised studies of interventions. BMJ 2016;355:i4919.

24 Sterne JAC, Higgins JPT, Reeves BC. A Cochrane risk of bias assessment tool: for Non-Randomized studies of interventions (ACROBAT-NRSI), version 1.0.0, 2014. Available: http://www.bristol. ac.uk/population-health-sciences/centres/cresyda/barr/riskofbias/ robins-i/acrobat-nrsi/ [Accessed 1 Jul 2019].

25 Hayden JA, van der Windt DA, Cartwright JL, et al. Assessing bias in studies of prognostic factors. Ann Intern Med 2013;158:280-6.

26 Munn Z, Peters MDJ, Stern C, et al. Systematic review or scoping review? Guidance for authors when choosing between a systematic or scoping review approach. BMC Med Res Methodol 2018:18:143-43.

27 Miake-Lye IM, Hempel S, Shanman R, et al. What is an evidence MAP? A systematic review of published evidence maps and their definitions, methods, and products. Syst Rev 2016;5:28.

28 Dias S, Sutton AJ, Ades AE, et al. Evidence synthesis for decision making 2: a generalized linear modeling framework for pairwise and network meta-analysis of randomized controlled trials. Med Decis Making 2013;33:607-17.

29 Stijnen T, Hamza TH, Ozdemir P. Random effects meta-analysis of event outcome in the framework of the generalized linear mixed model with applications in sparse data. Stat Med 2010;29:3046-67.

30 Higgins JPT, Thompson SG, Deeks JJ, et al. Measuring inconsistency in meta-analyses. BMJ 2003;327:557-60.

31 Sterne JAC, Sutton AJ, loannidis JPA, et al. Recommendations for examining and interpreting funnel plot asymmetry in meta-analyses of randomised controlled trials. BMJ 2011;343:d4002.

32 Turner NL, Dias S, Ades AE, et al. A Bayesian framework to account for uncertainty due to missing binary outcome data in pairwise metaanalysis. Stat Med 2015;34:2062-80.

33 Guyatt GH, Oxman AD, Vist GE, et al. Grade: an emerging consensus on rating quality of evidence and strength of recommendations. $B M J$ 2008:336:924-6.

34 Morzycki A, Bhatia A, Murphy KJ. Adverse reactions to contrast material: a Canadian update. Can Assoc Radiol J 2017:68:187-93.

35 The Japan Radiological Society. Revised recommendations on steroid premedication for reducing the risk of acute adverse effects of iodinated contrast agents and gadolinium contrast agents, 2018 Available: http://www.radiology.jp/member_info/safty/20181115.html [Accessed 1 Jul 2019]. 